\title{
Lymphoepithelioma-like Carcinoma of the Breast: A Case Report
}

Ömer Erdinç Top ${ }^{1}$, Enver Vardar ${ }^{1}$, Ayşe Yağcl ${ }^{1}$, Senem Deniz ${ }^{1}$, Rafet Öztürk ${ }^{1}$, Baha Zengel ${ }^{2}$

${ }^{1}$ Department of Pathology, İzmir Bozyaka Training and Research Hospital, İzmir, Turkey

${ }^{2}$ Department of General Surgery, İzmir Bozyaka Training and Research Hospital, İzmir, Turkey

\begin{abstract}
Lymphoepithelioma-like carcinoma carries similar histopathological features with lymphoepithelioma typically located in the nasopharynx. Lymphoepithelioma-like carcinoma of the breast can be mistaken for breast lymphoma or medullary carcinoma due to the undifferentiated appearance of tumor cells and presence of prominent lymphoid component. Lymphoepithelioma-like carcinoma is rare, and the similarity between medullary carcinoma of the breast makes it difficult to distinguish these two tumors. In the presented case, neither lymph node nor distant metastases were detected. Breast lymphoepithelioma-like carcinoma is extremely rare with only 21 reported cases in the literature. Herein we present a 59-year-old woman with lymphoepithelioma-like carcinoma of the breast along with the cases previously published in the literature.
\end{abstract}

Key words: Breast carcinoma, lymphoepithelioma-like carcinoma, lymphoepithelioma

\section{Introduction}

Regaud and Schminke first described lymphoepithelioma-like carcinoma (LELC) in 1921, as an undifferentiated carcinoma accompanied by prominent lymphocytic infiltration and located in the nasopharynx $(1,2)$. Schminke and Regaud have identified the patterns in which malignant cells are present either in isolation (Schminke's pattern) or syncytial masses, nests or islands (Regaud's pattern) (3). Kumar et al. (4) have reported the first LELC of the breast in 1994. LELC of the breast has been described as carrying similar morphological features with undifferentiated nasopharyngeal carcinoma (1-5). A total of 21 breast LELC cases have been reported in the literature, thus our patient is presented together with these reports due to its rarity.

\section{Case Presentations}

A 59 -year-old female patient had a $3 \mathrm{~cm}$ mass on her breast for the last 2 months, which proved to be malignant on biopsy. She had undergone a right modified mastectomy (MRM) in another center. The macroscopic examination of the material there showed a solid, gray-white tumor of $2.5 \mathrm{~cm}$ in diameter that was located in the upper outer quadrant of the breast. She was referred to our clinic for further evaluation and treatment. The re-evaluation of the specimen's paraffin blocks revealed large areas of abundant lymphocytic mononuclear inflammatory cells and non- cohesive tumor cells in the form of either individual cells or, rarely, small groups located within fibrous stromal connective tissue (Figure 1). Acidophilic cytoplasm with eccentric, hyperchromatic nuclei and rare nucleoli were observed. Non- neoplastic cells in lymphoid nature were detected to be dispersed in intratumoral and peritumoral areas. Tubulus or ductus formation was not observed in tumor sections. Lobular or ductal carcinoma in situ and lobular syncytial growth pattern were not detected in the surrounding breast parenchyma. The tumor contained extensive areas of necrosis as well as areas of sclerosing lymphocytic lobulitis within the surrounding breast parenchyma.

In immunohistochemistry tumor cells showed diffuse positivity with pancytokeratin (Figure 2) and CK7, and focal mild positivity with e-cadherin, the Ki67 (MIB1) proliferation index was 7\%. The results of other immunohistochemical stains are given in Table 1. In lymphoid cells, diffuse staining was observed with CD3, and focal staining with CD20 (Figure 3). In addition, CD8 and CD4 positive cells in lymphoid cells were in similar proportions. Balanced staining was seen with kappa and lambda. Metastasis was not observed in 23 lymph nodes dissected from the axilla. Based on the histopathological and immunohistochemical findings the case has been reported as "lymphoepithelioma-like carcinoma". Following the primary fluorouracil, epirubicin, cyclophosphamide (FEC) protocol, docetaxel trihydrate treatment was applied, and she is being followed-up for 9 months without any evidence of toxicity. 


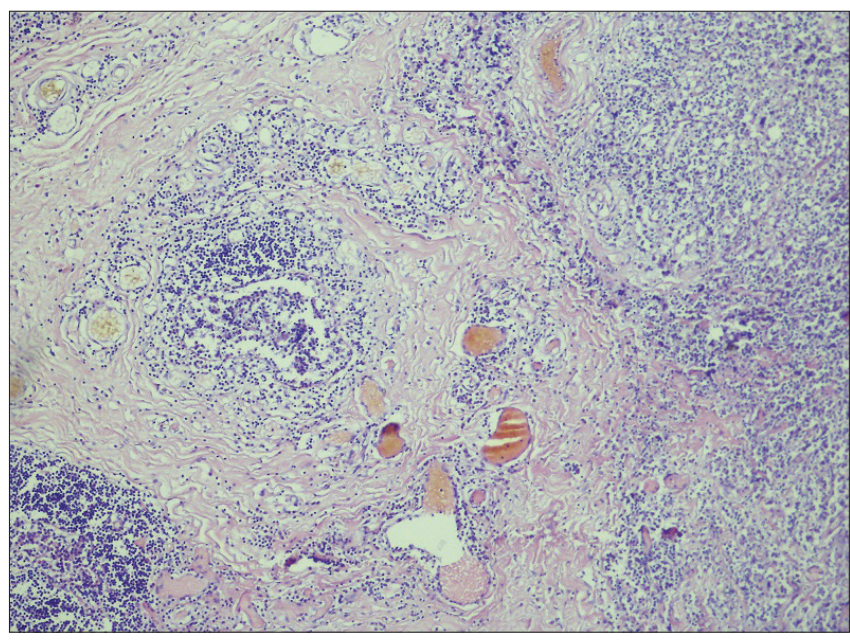

Figure 1. Scattered tumor cells with acidophilic cytoplasm within abundant lymphocytic component (x200-HE)

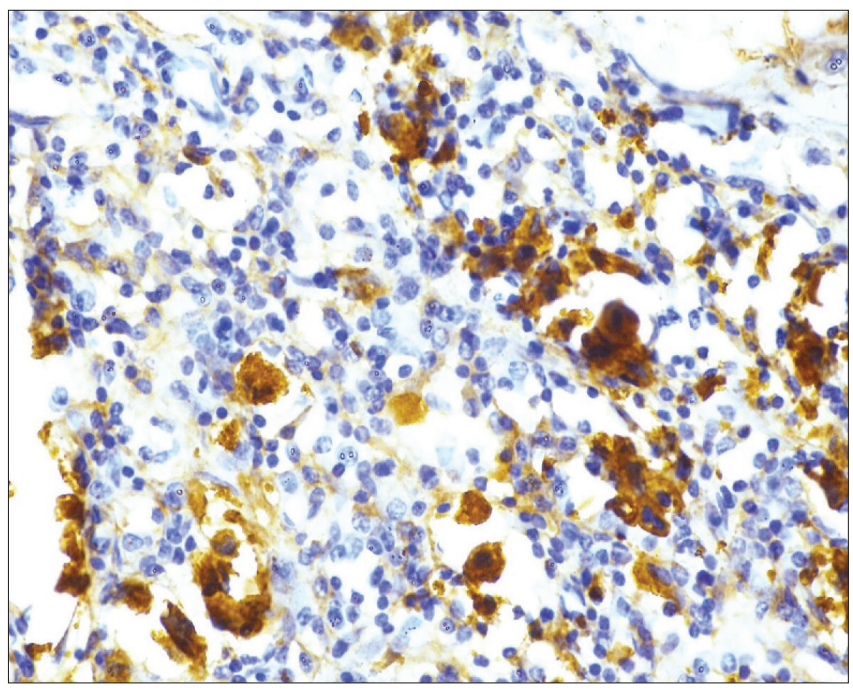

Figure 2. IHC cytokeratin dye making clearer the hardly noticed epithelial component among cells with lymphoid characteristics (x200-pansitokeratin)

\section{Discussion and Conclusions}

Lymphoepithelioma is a carcinoma with undifferentiated cytomorphological features, and was first identified in the nasopharynx, however its presence in multiple other organs was later reported $(1,6)$. LELC is used synonymously with lymphoepithelioma and lymphoepithelial carcinoma (6). On the other hand, some authors advocate that LELC should be used in tumors with similar morphological features and in those located outside the nasopharynx (7). Cases in locations such as the skin, lacrimal and salivary glands, thyroid, thymus, lung, esophagus, stomach, colon, hepatobiliary system, renal pelvis, ureter, kidney, bladder, prostate, cervix, vulva and vagina have also been reported (1).

The basic morphological features in breast LELC first reported by $\mathrm{Ku}$ mar et al (4) in 1994 is the presence of neoplastic epithelial cells in small clusters or isolated scattered cells showing minimal atypia with eosinophilic cytoplasm accompanied by significant lymphocytes. Essentially two histological patterns are present in these tumors: "Regaud pattern" and "Schminke pattern", and it is reported that distinguishing
Table 1. Hormone receptor status and cerbB-2 results of all reported cases in the literature

\begin{tabular}{lcccc} 
& \multicolumn{4}{c}{ Results of all cases } \\
& Positive & Negative & Indeterminate & Total \\
Estrogen & 10 & 8 & 1 & 19 \\
Progesterone & 12 & 5 & 2 & 19 \\
cerbB-2 & 11 & 2 & 5 & 19
\end{tabular}

cance. However, recognizing these patterns and distinction from breast carcinomas and lymphoma containing dense lymphocytic infiltrate is important $(1,6,8)$. In the case presented, "Schminke pattern" was observed in almost all areas.

The differential diagnosis of lymphoepithelioma-like carcinoma (LELC) should include medullary carcinoma, and the less likely ductal or lobular carcinomas accompanied by prominent lymphoid component $(5,7)$. The presence of dominant Regaud pattern accompanied by intense lymphoid cell infiltration, in other words breast LELC with prominent syncytial growth pattern, may be especially confused with medullary carcinoma. (9). The typical smooth peripheral boundaries of medullary carcinoma in macroscopy, and the absence of infiltration in boundaries in microscopy are important features for distinction. In our case, the dissection was not carried out by our department therefore we were unable to evaluate the macroscopic limits, however microscopically significant infiltrative pattern was observed. In medullary carcinoma, in addition to the limited appearance the syncytial growth pattern consisting of cells with a high mitotic activity should be seen in more than $75 \%$ of the microscopic field. (9). In our case, the syncytial pattern was not observed. The lymphoid component of the stroma may be noteworthy in some invasive ductal or lobular breast carcinomas. But in this tumor the lymphoid component is less than that in LELC. In addition to these properties, the infiltration in ductal and lobular carcinoma form large islands, tubules or cribriform, comedocarcinoma growth pattern and they show significant stromal desmoplasia, further helping in distinguishing these tumors from LELC (1-3). In our case, duct -like structures in the tumor or areas of ductal carcinoma in situ was not found.

Non-Hodgkin's lymphoma should also be considered in the differential diagnosis (8). Lymphoma should not contain epithelial components, and in cases where distinction between epithelial cells and mononuclear lymphoid cells is difficult, tumor cells in epithelial nature can be detected among dense lymphoid cells by immunohistochemical evaluation for EMA and cytokeratin (1-3, 5, 8, 9).

Although a strong relationship between Epstein- Barr virus (EBV), which plays an important role in the pathogenesis of nasopharyngeal lymphoepithelioma, and LELC has been defined, a relationship between breast LELC and EBV has not been shown in the literature (2-5, 9, 10). In our case, EBV was found to be negative. The sclerosing lymphocytic lobulitis morphology that has been previously defined in only one case report was also observed in our case, in the area surrounding the tumor. It is proposed that both sclerosing lymphocytic lobulitis and LELC might be triggered by the same immunologic factors. On the other hand, it has also been reported that sclerosing lymphocytic lobulitis may be a response against the tumor (2). Naidoo et al. (2) reported that the dominant lymphoid component was B-lymphocytes within the tumor, while T-lymphocytes were dominant in sclerosing 


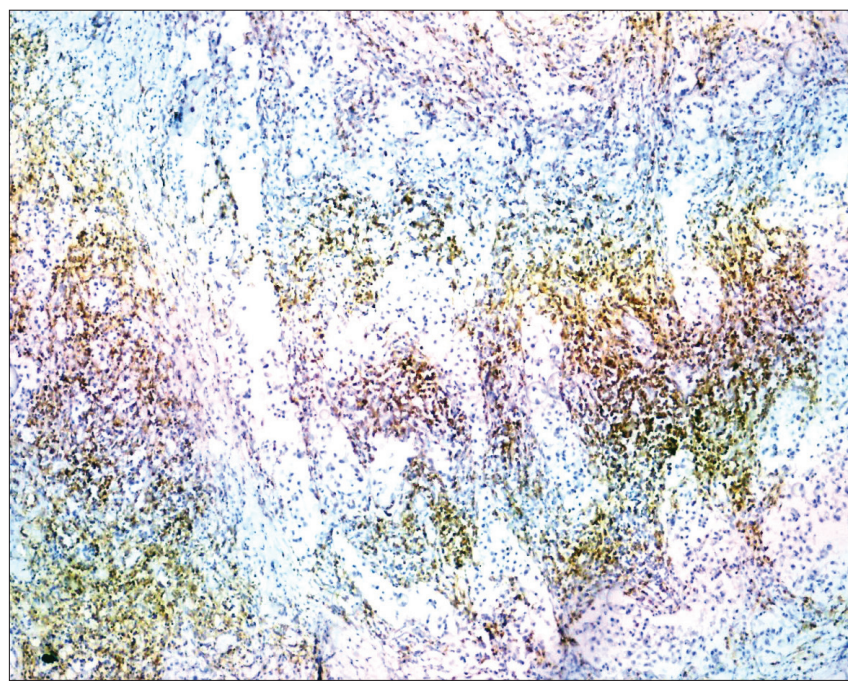

Figure 3. $\mathrm{CD} 3$ positive $T$ lymphocytes in groups that contain vague epithelial component $(\times 100-C D 3)$

\section{Table 2. Immunohistochemistry results}

\begin{tabular}{lcc} 
Antibody & Clone/Brand & Result \\
\hline ER & ID5/Thermo & $(-)$ \\
PR & PgR636/Dako & $(-)$ \\
cerbB-2 & Poliklonal/Dako & $(-)$ \\
e-cadherin & NCH-38/Dako & Focal weak (+) \\
Ki67 & MIB1/Dako & $\% 7$ \\
P53 & DO-7/Thermo & Focal weak (+) \\
EBV & CS.1-4/Dako & $(-)$ \\
HMWK & 34Be12/Genemed & $(-)$ \\
CD117 & Polyclonal/Dako & $(-)$ \\
GCDFP15 & 23A3/Thermo & $(-)$ \\
CK5/6 & D5/16 B4/Dako & $(-)$ \\
EGFR & EGFR.25/Novocastra & $(-)$ \\
HMB45 & HMB45/Dako & $(-)$ \\
CD10 & 56C6/Novocastra & $(-)$ \\
Vimentin & Vim3B4/Dako & $(-)$
\end{tabular}

ER: Estrogen receptor; PR: Progesterone receptor; EBV: Epstein-Barr virus; HMWK: High molecule weight keratin; GCDFP15: Gross cystic disease fluid protein-15; CK5/6: Cytokeratin 5/6; EGFR: Epidermal growth factor receptor; HMB45: Human Melanoma Black45

lobulitis areas. In our case, T-lymphocytes were dominant both within the tumor and in areas of lobulitis.

In 5 of the 21 cases reported in the literature, axillary lymph node metastasis was identified, in our patient metastases was not observed in 23 lymph nodes dissected from the axilla (1, 11-14). Immunohistochemical staining results of 19 patients out of 21 reported cases in the literature are given in Table 2. HER2 was negative in 11 of 13 patients who had this staining. Seven cases who were ER (-), PR (-), and cerbB2-negative (triple-negative) have been reported, as was our case $(1,13,14)$. One case who was diagnosed with LELC by fine needle aspiration biopsy has also been reported (12).
In conclusion, the meta-analysis of hormone receptor and cerbB -2 immunoreactivity results of 19 cases out of the 21 reported breast LELC patients showed 7 triple- negative cases, and 11 of 13 patients $(84.6 \%)$ who had cerbB -2 assesment was reported to be negative $(1,13,14)$. In one patient the hormone receptor -positivity criterion was defined as the presence of staining in at least $10 \%$ of cells (10). Therefore, it may be concluded that comparison of the rates of hormone receptor status in breast LELC and overall rates in breast tumors might not be reliable. Since different experts have evaluated the cases reported in the literature at different times, and immunohistochemical staining techniques differ due to subjective elements, the rates of hormone receptor status are likely to become more accurate as the number of reported cases and case series increase. When analyzed in terms of the molecular classification of breast tumors, it can be stated that almost all of these tumors are cerbB-2 negative and unlike the one located in the nasopharynx they are EBV-negative.

Breast LELC is a rare tumor with characteristic pathologic properties, which may result in lymph node or distant metastasis. Breast-conserving surgery is recommended in selected cases. The role of systemic chemotherapy and radiation therapy is not clear except in cases of metastases, and molecular targeted therapy may be considered due to the positivity of hormone receptors, however they are usually cerbB-2 negative. Long-term follow-up results are not known. Although their prognosis is a relatively better, it is worse than that of medullary carcinoma, and therefore they should be considered in the differential diagnosis.

Conflict of Interest: No conflict of interest was declared by the authors.

Peer-review: Externally peer-reviewed.

Informed Consent: Written informed consent was obtained from patients who participated in this case.

Author Contributions: Concept - Ö.E.T.; Design - Ö.E.T.; Supervision - E. V.; Funding - E.V.; Materials - B.Z.; Data Collection and/ or Processing - A.Y.; Analysis and/or Interpretation - R.Ö.; Literature Review - S.D.; Writer - Ö.E.T.; Critical Review - E.V.

Financial Disclosure: No financial disclosure was declared by the authors.

\section{References}

1. Dinniwell R, Hanna WM, Mashhour M, Saad RS, Czarnota GJ. Lymphoepithelioma-likec arcinoma of the breast: a diagnostic and therapeutic challenge. Curr Oncol 2012; 19:177-183. (PMID: 22670107) [CrossRef]

2. Naidoo P, Chetty R. Lymphoepithelioma-like carcinoma of the breast with associated sclerosing lymphocytic lobulitis. Arch Pathol Lab Med 2001; 125:669-672. (PMID: 11300942)

3. Peştereli HE, Erdogan O, Kaya R, Karaveli FS. Lymphoepithelioma-like carcinoma of the breast. APMIS 2002; 110:447-450. (PMID: 12193205 [CrossRef])

4. Kumar S, Kumar D. Lymphoepithelioma-like carcinoma of the breast. Mod Pathol 1994; 7:129-131. (PMID: 7512716)

5. Ilvan S, Celik V, Ulker Akyildiz E, Senel Bese N, Ramazanoglu R, Calay Z. Lymphoepithelioma-like carcinoma of the breast: is it a distinct entity? Clinicopathological evaluation of two cases and review of the literature. Breast 2004; 13:522-526. (PMID: 15563863) [CrossRef]

6. Barnes L, Eveson JW, Reichart P. WHO histological classification of tumours of the nasopharynx. World Health Organization Classification of 
Tumours: Pathology and Genetics of Head and Neck Tumours. Lyon, France: IARC Press, 2005; 81-106.

7. Kurose A, Ichinohasama R, Kanno H, Kobayashi T, Ishida M, Nishinari $\mathrm{N}$, Sawai T. Lymphoepithelioma-like carcinoma of the breast. Report of a case with the first electron microscopic study and review of the literature. Virchows Arch 2005; 447:653-659. (PMID: 15947950) [CrossRef]

8. Vardar E, Ozkok G, Cetinel M, Postaci. H. Primary breast lymphoma cytologic diagnosis. Arch Pathol Lab Med 2005; 129:694-696. (PMID: 15859646)

9. Dadmanesh F, Peterse JL, Sapino A, Fonelli A, Eusebi V. Lymphoepithelioma-like carcinoma of the breast: lack of evidence of Epstein-Barr virus infection. Histopathology 2001; 38:54-61. (PMID: 11135047) [CrossRef]

10. Cristina S, Boldorini R, Brustia F, Monga G. Lymphoepithelioma-like carcinoma of the breast: an unusual pattern of infiltrating lobular carcinoma. Virchows Arch 2000; 437:198-202. (PMID: 10993283) [CrossRef]
11. Nio Y, Tsuboi K, Tamaoki M, Tamaoki M, Maruyama R. Lymphoepithelioma-like carcinoma of the breast: a case report with a special analysis of an association with human papilloma virus. Anticancer Res 2012; 32:1435-1441. (PMID: 22493382)

12. Trihia H, Siatra H, Gklisty H, Diamantopoulos P, Arapantoni-Dadiotis P, Kalogerakos K. Lymphoepithelioma-like carcinoma of the breast: cytological and histological features and review of the literature. Acta Cytol 2012; 56:85-91. (PMID: 22236751) [CrossRef]

13. O'Sullivan-Mejia E, Idowu MO, Davis Masssey H, Cardenosa G, Grimes MM. Lymphoepithelioma-like carcinoma of the breast: diagnosis by core needle biopsy. Breast J 2009; 15:658-660. (PMID: 19824996) [CrossRef]

14. Jeong AK, Park SB, Kim YM, Ko BK, Yang MJ, Kwon WJ, Lee JH, Weon YC. Lymphoepithelioma-like carcinoma of the breast. J Ultrasound Med 2010; 29:485-488. (PMID: 20194945) 